\title{
Association between Lipid Profile Components and Ischemic Cerebrovascular Disease
}

\author{
Abhishek Kumar Chandra', Anurag Kumar², Ansuman Dalbehera ${ }^{3}$, \\ Prakash Chandra Singh ${ }^{4}$
}

\author{
${ }^{1}$ Associate Professor, Department of Medicine, Gauri Devi Institute of Medical Science, Durgapur, West Bengal \\ ${ }^{2}$ Assistant Professor, Traumatic and Emergency, General Surgery, AIIMS, Patna, Bihar \\ ${ }^{3}$ Senior Consultant Dept of Medicine, Batra Hospital Medical Research Centre, New Delhi \\ ${ }^{4}$ Associate Professor, Department of Paediatrics Gauri Devi Institute of Medical Science, Durgapur (WB)
}

Corresponding Author: Abhishek Kumar Chandra

\begin{abstract}
Objective: The main objective of the study was to evaluate the association between lipid profile components and Ischemic Cerebrovascular Disease.

Materials and Methods: This was an observational retrospective study done in a tertiary care hospital. A pre designed pro forma were prepared to capture the required data for their clinical records. Before enrolment a verbal consent were taken from all the participants after thoroughly discussed the objective and purpose of the study with them. Records of clinical examination, CT scan brain and other serum investigation reports were noted for further analysis.
\end{abstract}

Results: Total 140 patients whose average mean age was $65.3 \pm 9.1$ years were participated in this study. Among all the participants $60 \%$ were male. Participants were having west circumference of $37.8 \pm 5.2 \mathrm{~cm}$. Among the participants $68 \%$ were having hypertension, $23 \%$ were diagnosed previously with diabetes, $16 \%$ having current smoking history and $17 \%$ were moderately consuming alcohol. Among the total participants 70 were belongs to study groups and rest 70 were belongs to control groups. PCA was involved in 34\%, MCA was involved in 54\% and ACA was involved in $12 \%$ of cases. Total 19 participants were having hyperlipidemia among which 14 (73.7\%) were belongs to IIIb groups, 3 (15.8\%) were belongs to IIa group and $2(10.5 \%)$ were belongs to IV group. Among total participants who were diagnosed with having elevated hyperlipidemia, 7 were male and 12 were female.
Conclusion: The main goal should be rather than treatment to target the presentation of atherosclerosis. As per latest available guidelines and based on the calculated risk drug treatment for hyperlipidemia should be individualised.

Keywords: Hyperlipidemia, Stroke, Ischemic cerebro vascular disease.

\section{INTRODUCTION}

Stroke is the second commonest cause of death and disability in the developing world. The crude prevalence rate of stroke in India can be as high as 942/100,000 population, although the pooled data suggested a more moderate prevalence rate of $115-203 / 100,000$ and the incidence from 105-124/100,000. Prevention is of utmost importance in lowering the public health impact of stroke, especially because of high incidence. People at risk need to be identified in order to institute these stroke prevention strategies [1]. Some risk factors, such as age, sex, race-ethnicity and heredity are not modifiable. These serve as markers for those at high risk. However, there are also multiple modifiable risk factors with numerous options for intervention [2].

Dyslipidaemia is refer to the rearrangements of one or many of the lipoproteins; elevations of total cholesterol, low density lipoprotein (LDL) cholesterol and/or triglycerides white elevation of 
lipoproteins alone is labels as "hyperlipidaemia". Exogeneous pathway begins with intestinal incorporation of dietary triglycerides (TG) and cholesterol into large lipoprotein particles called chylomicrons (diameter 80-500 nm) [3]. They enter the blood stream via lymph and are acted on by lipoprotein lipase existing on the endothelial cells of the capillaries in adipose tissue and muscles. Fatty acids (FA) liberated from TG enter the cells and get reconverted to TG or used as fuel. Primary dyslipidaemia (e.g. Familial hypercholesterolemia) typically refers to a genetic defect in the lipid metabolism that causes abnormal lipid levels [4]. A secondary dyslipidaemia may be due to a variety of reasons; environmental factors (diet rich in saturated fat or a secondary lifestyle), diseases (type 2 diabetes, hypothyroidism; obstructive jaundice etc.) and medications (thiazide diuretics, progestins, anabolic steroids etc). Secondary dyslipidaemias could be corrected or ameliorated by treating the underlaying disorders [5].

Recent clinical trials suggest that stroke may be reduced by the administration of statin agents in persons with CAD [6-8]. The risk reductions with statins were beyond that expected solely through cholesterol reductions and have led to the consideration of other potential beneficial mechanisms. These findings led to approval of statin for the prevention of stroke in those with CAD. The main objective of the study was to evaluate the association between lipid profile components and Ischemic Cerebrovascular Disease.

\section{MATERIALS AND METHODS}

This was an observational retrospective study done in a multimeter terry care hospitals. A pre designed pro forma were prepared to capture the required data for their clinical records. Before enrolment a verbal consent were taken from all the participants after thoroughly discussed the objective and purpose of the study with them.
Main inclusion criteria of these study was patients with focal neurological disturbance with relative or abrupt rapid onset which persisted for more than 24 hours or stuttering with smooth progression over few hours and CT scan evidence. The main exclusion criteria were hematological disturbances, pregnancy, with head injury, hepatic encephalopathy and with previous neurological deficits and TIAs.

Records of clinical examination, CT scan brain and other serum investigation reports were noted for further analysis. For lipid parameters following serum test reports were fetched for further analysis i.e.; Serum total cholesterol (TC), Serum triglycerides (TG), High density lipoprotein (HDL), and Low density lipoprotein (LDL).

To analyse data for this study we combined both descriptive and inferential statistics. We made use of simple percentages among the descriptive statistics along with mean and standard deviation. At 0.05 level of significance, we made use of hierarchical multiple regression and $t$-test. With the use of Statistical Package for Social Sciences (SPSS) version 22, all the analyses were done.

\section{RESULTS}

Total 140 patients whose average mean age was $65.3 \pm 9.1$ years were participated in this study. Among all the participants $60 \%$ were male. Participants were having west circumference of $37.8 \pm 5.2$ $\mathrm{cm}$. Among the participants $68 \%$ were having hypertension, $23 \%$ were diagnosed previously with diabetes, $16 \%$ having current smoking history and $17 \%$ were moderately consuming alcohol. All demographic characteristics were illustrated in table 1 .

Table 1: Demographic characteristics of the participants
\begin{tabular}{|l|c|}
\hline Characteristics & $(\mathbf{N}=140)$ \\
\hline Age (Years) & $65.3 \pm 9.1$ \\
\hline Male (N\%) & $84(60 \%)$ \\
\hline Risk factors & $95(68 \%)$ \\
\hline Hypertension & $32(23 \%)$ \\
\hline Diabetes mellitus & $22(16 \%)$ \\
\hline Current smoking & $37.8 \pm 5.2$ \\
\hline Wais circumference & $24(17 \%)$ \\
\hline Moderate alcohol intake &
\end{tabular}


Among the total participants 70 were belongs to study groups and rest 70 were belongs to control groups. PCA was involved in $34 \%$, MCA was involved in
$54 \%$ and ACA was involved in $12 \%$ of cases. Lipid Profile in study and control groups were depicted in table 2 .

Table 2: Lipid Profile in study and control groups

\begin{tabular}{|l|c|c|c|c|}
\hline \multicolumn{1}{|c|}{ Parameters } & Study Group (N=70) & Control Group $(\mathbf{N}=70)$ & T value & P Value \\
\hline Total Cholesterol (TC) $(\mathrm{mg} / \mathrm{dl})$ & $210.8 \pm 43.6$ & $142.6 \pm 19.2$ & 6.893 & $<0.01$ \\
\hline Triglycerides (TG) $(\mathrm{mg} / \mathrm{dl})$ & $183.2 \pm 46.9$ & $129.7 \pm 11.5$ & 4.854 & $<0.01$ \\
\hline Low density Lipoprotein (LDL) (mg/dl) & $142.7 \pm 32.3$ & $84.1 \pm 17.2$ & 7.691 & $<0.01$ \\
\hline High Density Lipoprotein (HDL)(mg/dl) & $33.6 \pm 5.1$ & $46.4 \pm 2.8$ & 11.538 & $<0.01$ \\
\hline
\end{tabular}

Table 3 shows incidence of hyperlipidemia types. Total 19 participants were having hyperlipidemia among which $14(73.7 \%)$ were belongs to IIIb groups, 3 $(15.8 \%)$ were belongs to IIa group and 2 $(10.5 \%)$ were belongs to IV group. Among total participants who were diagnosed with having elevated hyperlipidemia, 7 were male and 12 were female.

Table 3: Types of Hyperlipidemia

\begin{tabular}{|c|c|c|c|c|}
\hline $\begin{array}{c}\text { Type of } \\
\text { Hyperlipidemia }\end{array}$ & Male & Female & Total & Percentage \\
\hline IIIb & 6 & 8 & 14 & $73.7 \%$ \\
\hline IIa & 1 & 2 & 3 & $15.8 \%$ \\
\hline IV & & 2 & 2 & 10.5 \\
\hline Total & $\mathbf{7}$ & $\mathbf{1 2}$ & $\mathbf{1 9}$ & $\mathbf{1 0 0 \%}$ \\
\hline
\end{tabular}

\section{DISCUSSION}

A review of recent prevention guidelines concerning cholesterol lowering by statin use in stroke prevention suggested that the national Cholesterol Education Program (NCEP), Expart Panel on Detection, Evaluation and Treatment of High Cholesterol in Adults (Adults Treatment Panel III), is the most comprehensive guide for the management of lipids in persons at risk for or who have cerebrovascular disease. NCEP emphasises LDL-Cholesterol lowering and 2 major modalities for LDL-C lowering: therapeutic life style change and drug specific therapy. When there is a history of CAD and CAD risk equivalents, the target LDL-C goal is $<100 \mathrm{mg} \%$. LDL-C lowering results in a reduction of total mortality, coronary mortality, major coronary events, coronary procedures and stroke in patients with CAD. The recommendation in very high risk persons is to aim for an LDL-C of $<70$ $\mathrm{mg} \%$.
In landmark Scandinavian Simvastatin Survival Study (4S) [9] proved a watershed in the debate concerting the safety and rationale of cholesterol lowering therapy. Patients with established CHD $(\mathrm{N}=4444)$ were followed up for 5 years with and without cholesterol lowering. Reduction in TC and LDL-C of $25 \%$ and $35 \%$ respectively was associated with a $42 \%$ reduction in the risk of cardiovascular mortality and a 30\% reduction in total mortality. Subsequent analysis and the publication of two other secondary prevention trials CARE [10] and LIPID [11] (both using pravastatin) have confirmed reductions in mortality, coronary events, subsequent revascularization, hospital stay and development of angina, heart failure, stroke and intermittent claudication. Enin in current study total 19 participants were having hyperlipidemia among which 14 (73.7\%) were belongs to IIIb groups, 3 $(15.8 \%)$ were belongs to IIa group and 2 $(10.5 \%)$ were belongs to IV group.

Very high risk patients are those who have established cardiovascular disease plus (1) Multiple major risk factors (especially diabetes mellitus); (2) severe and poorly controlled risk factors (especially continues cigarette smoking); (3) Multiple risk factors of the metabolic syndrome (especially high triglycerides $\geq 200 \mathrm{mg} / \mathrm{dl}$ with low HDL cholesterol $(\leq 40 \mathrm{mg} / \mathrm{dl})$ and (4) Patients with acute coronary syndromes [12]. Even in current study among the participants $68 \%$ were having hypertension, $23 \%$ were diagnosed previously with diabetes, $16 \%$ having current smoking history and $17 \%$ were moderately consuming alcohol. 
In cases of ICD than in controls there were significantly higher serum triglyceride levels. These observations were in line with the previous studies. Symptomatic intracranial atherosclerotic disease was associated with High TG: HDL$\mathrm{C}$ ratio and for optimizing intensive medical treatment may represent a therapeutic target [13]. Our observation also in line with and confirms low HDL-cholesterol in patients with dyslipidemia support the use of therapeutic strategies to counter the common finding [14].

\section{CONCLUSION}

The main goal should be rather than treatment to target the presentation of atherosclerosis. As per latest available guidelines and based on the calculated risk drug treatment for hyperlipidemia should be individualised.

\section{Acknowledgement: None}

\section{Conflict of Interest: None}

\section{Source of Funding: None}

\section{Ethical Approval: Approved}

\section{REFERENCES}

1. Wilson PW, Anderson KM, Harris T, Kannel WB, Castelli WP: Determinants of change in total cholesterol and HDL-C with age: the Framingham Study. J Gerontol 1994;49:M252-M257.

2. Debette S, Beiser A, DeCarli C, Au R, Himali JJ, Kelly-Hayes M, Romero JR, Kase CS, Wolf PA, Seshadri S: Association of MRI markers of vascular brain injury with incident stroke, mild cognitive impairment, dementia, and mortality: the Framingham Offspring Study. Stroke 2010; 41:600-606.

3. Cholesterol, diastolic blood pressure, and stroke: 13,000 strokes in 450,000 people in 45 prospective cohorts. Prospective studies collaboration. Lancet 1995;346: 1647-1653.

4. Amarenco P, Bogousslavsky J, Callahan A 3rd, Goldstein LB, Hennerici M,
Rudolph AE, Sillesen H, Simunovic L, Szarek M, Welch KM, et al: High-dose atorvastatin after stroke or transient ischemic attack. N Engl J Med 2006; 355:549-559.

5. Jimenez-Conde J, Biffi A, Rahman R, Kanakis A, Butler C, Sonni S, Massasa E, Cloonan L, Gilson A, Capozzo K, et al: Hyperlipidemia and reduced white matter hyperintensity volume in patients with ischemic stroke. Stroke 2010;41:437-442.

6. Kurth T, Everett BM, Buring JE, Kase CS, Ridker PM, Gaziano JM. Lipid levels and the risk of ischemic stroke in women. Neurology. 2007;68(8):556-562.

7. Bhattacharya S, Prasarsaha S, Basu A, Das K. A 5 year prospective study of incidence, morbidity and mortality stroke profile on stroke in a rural community of Eastern India. J Indian Med Assoc 2005; 103(12): 655-9.

8. Hindy G, Engström G, Larsson SC, Traylor M, Markus HS, Melander O, et al.; Stroke Genetics Network (SiGN). Role of blood lipids in the development of ischemic stroke and its subtypes: a mendelian randomization study.Stroke. 2018; 49:820-827.

9. Mackness M, Arrol S, Abbott $\mathrm{C}$, et al. (1993) Protection of LDL against oxidative modification by HDL associated paraoxonase. Atherosclerosis 104:129135.

10. Scandinavian Simvastatin Survival Study Group. Randomised trial of cholesterol lowering in 4444 patients with coronary heart disease: the Scandinavian Simvastatin Survival Study (4S). Lancet 1994;344:1383.

11. Sacks FM, Moye LA, et al. Relationship between plasma LDL concentration during treatment with pravastatin and recurrent coronary events in the Cholesterol and Recurrent Events trial. Circulation 1998:97: 1446.

12. The Long Term intervention with Pravastatin in Ischemic Disease Study Group. Prevention of cardiovascular events and death with pravastatin in patients with coronary heart disease and a broad range of initial cholesterol levels. $\mathrm{N}$ Engl J Med. 1998;339:1349-1357. 
13. Vishal B Jani, Saqib S Chaudhry, Vikram Jadhav, Sayed Hussain, Adnan Safdar, and Adnan I Qureshi. Association of Triglyceride-HDL Cholesterol and LDL Cholesterol HDL Cholesterol Ratios With Symptomatic Intracranial Atherosclerosis. Stroke. 2014; 45:AWP338. DOI: https://doi.org/10.1161/str.45.suppl_1.wp3 38

14. Philip Barter. The role of HDL-cholesterol in preventing atherosclerotic disease.
European Heart Journal Supplements (2005) 7 (Supplement F), F4-F8. DOI: 10.1093/ eurheartj/sui036.

How to cite this article: Chandra AK, Kumar A, Dalbehera A et.al. Association between lipid profile components and ischemic cerebrovascular disease. International Journal of Research and Review. 2021; 8(7): 486-490. DOI: https://doi.org/10.52403/ijrr.20210769 